\title{
Die Nitiduliden Japans.
}

\section{Von Edm. Reitter in Mödling.}

(Fortsetzung. Vide pag. 44.)

\section{Pocadites dilatimanus Reitt. (Stelidota.)}

Bei Beschreibung dieser Art lag mir blos ein von Hiller gesammeltes, nicht schön erhaltenes Stück vor, bei dem die Behaarung nicht deutlich sichtbar war. Diese ist auf Kopf und Halsschild gelb und schwarz marmorirt, auf den Flügeldecken bildet die gelbe, helle Behaarung zwischen der dunklen mehr oder minder deutliche Querbinden.

Einige kleinere, mehr eiförmige Stücke gehören vielleicht einer anderen Art an; sie sind schwarz, Fühler, Beine, die Ränder des Halsschildes und die gemeinschaftliche Scheibe der Flügeldecken rostroth. Ich habe sie durch den Namen dorsiger separirt.

Konose, Yuyama, Kashiwagi.

\section{Pocadites rufo-basalis:}

Ovatus, valde convexus, subopacus, subtiliter pubescens, antennarum basi tarsisque rufo-ferrugineis, capite (obscure castaneo) prothoraceque confertim, sat fortiter aequaliterque punctatis, hoc antrorsum angustato, transverso, antice emarginato, basi prope scutellum utrinque sinuato, lateribus tenuiter marginato, dorso aequaliter convexo, scutello nigro, dense punctato, elytris ovatis, dense punctatis, punctis fere seriatis, vix striatis, nigro-pubescentibus, fascia basali, extus parum abbreviata rubra et fulvo-puberula, lateribus breviter fulvociliatis, pygidio subnudo. Long. $5 \mathrm{~mm}$.

Von allen Arten durch die rostrothe Basalbinde der Flügeldecken, welche das schwarze Schildchen nicht einschliesst, und durch die robuste Körperform abweichend.

Ichiuchi, am 2. Mai 1881, ein Exemplar erbeutet.

\section{Pocadites oviformis:}

Oblongus, ovatus, leviter convexus, niger, nitidulus, nigro-, prothorace lateribus obsolete et elytrorum basi obsolete maculatim albido-puberulus, antennarum basi tarsisque ferrugineis, prothorace valde transverso, coleopteris haud angustiore, antrorsum valde angustato, dorso sat parce, lateribus crebre, fortiter punctato, antice emarginato, basi subrecta prope scutellum utrinque leviter sinuata, scutello fere semirotun-

„Wiener Entomologische Zeitung“ IV. (15. März 1885). Heft 3. 
dato, transverso, elytris dense punctatis, punctis seriatis, antice vix striatis, parce obsoletissime costulatis, costulis apicem versus distinctioribus, pygidio crebre fortiter punctato, subnudo. Long. $4 \mathrm{~mm}$.

Durch flacheren, länglich eiförmigen Körper recht bemerkenswerth.

Yuyama.

\section{Pocadites corpulentus:}

Breviter ovalis, valde convexus, niger, nitidus, antennarum basi ferruginea, tarsis rufis, capite thoraceque sat fortiter punctatis, pube nigra albidaque variegatis, prothoracis basi utrinque leviter bisinuata, in medio breviter prolongata, scutello fere semicirculari, parce punctato, elytris nigro-, basi maculatim albido-pubescentibus, seriatim sat fortiter sed minus profunde punctatis, interstitiis alternis seriatim nigropilosis. Long. $3 \cdot \tilde{\jmath}-4 \mathrm{~mm}$.

$\mathrm{Kukuwayama}$, am 3. Mai 1881 zahlreich erbeutet.

\section{Eugoniopus n. gen.}

Corpus ovale, convexum, glabrum. Labrum corneum, bilobum. Antennae breves, 11-articulatae, clava subrotundata triarticulata. Prothorax antice angustatus, basi immarginatus. Scutellum magnum, triangulare. Elytra striato-punctata, apice subtruncata, pygidium haud obtegentia. Prosternum pone coxas rotundatum, mesosternum occultum. Pedes robusti, tibiae apicem versus incrassatae, multicostatae, angulo externo apicali spinoso-producto. Tarsi anteriores sat dilatati, postici simplices. Diese neue Gattung hat viel Eigenthümliches; sie zeichnet sich vorzüglich durch die Schienenbildung aus. Die Schienen sind gegen die Spitze verbreitert, mit mehreren Längskanten versehen und ihre äussere Spitze ist dornförmig ausgezogen.

37. Eugoniopus Lewisi: (Jahrg. III, Taf. IV, Fig. 3.)

Miniato-rufus, maculis duabus prothoracis basalibus parvis, maculaque magna apicali in elytrorum angulo externo, nigris, antennarum clava fusca. Long. 7-9 $\mathrm{mm}$.

Minium- oder ziegelroth, zwei kleine viereckige Makeln an der Basis des Halsschildes und ein grösserer Flecken in der äusseren Apicalecke jeder Flügeldecke schwarz. Kopf klein, ungleichmässig punktirt, Clypeus eingeschnürt. Augen rund, fast glatt, schwarz, vortretend. Oberlippe gross, hornig, bis auf den Grund in zwei Lappen getheilt. Hals- 
schild an der Basis von der Breite der Flügeldecken, nach vorn verengt, vorn ausgerandet, mit den Flügeldecken in einer Flucht verlaufend, fein punktirt, dazwischen mit grösseren Punkten, welche an den Seiten zahlreicher sind, die Seiten fein erhaben gerandet, die Basis über das Schildchen etwas vortretend. Letzteres dreieckig, punktirt. Flügeldecken etwas länger als zusammen breit, mit dem Pygidium oval, mit ungefähr 10 wenig regelmässigen, kräftigen Punktreihen,

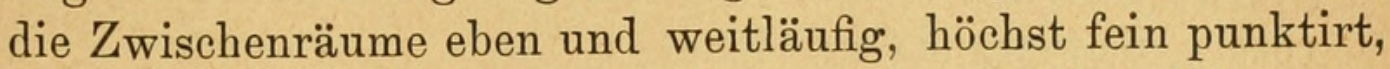
hinten abgestumpft oder fast abgestutzt, das Pygidium unbedeckt lassend. Letzteres dicht punktirt, an den Seiten gerandet. Das ôt hat ein sechstes kleines Analsegmentchen. Die Fühlerrinnen des Kopfes sind tief; um die Augen gebogen, nach hinten divergirend.

In einer Gattung Trüffeln, welche in dem Innern von grossen, hohlen, stark anbrüchigen Bäumen wachsen.

Oyayama, Nikko und Sapporo.

\section{Strongylus breviusculus:}

Subhemisphaericus, niger, nitidus, pedibus piceis, antennarum basiferruginea, tarsis rufis, elytris pygidium vix obtegentibus. Long. $2 \cdot 8-3 \mathrm{~mm}$.

Dem St. ater sehr ähnlich, aber doppelt kleiner, fast halbkugelig, also kürzer und stärker gewölbt, die Streifen der Flügeldecken deutlicher, die Zwischenräume derselben feiner punktirt, das Pygidium zum grössten Theile unbedeckt lassend.

Häufig ist der Körper, wie bei ater, einfärbig rostbraun. Miyanoshita, Idzu, im Mai 1881.

\section{Strongylus semiglobosus:}

Subhemisphaericus, niger, nitidus, antennarum basi tarsisque ferrugineis, elytris pygidium obtegentibus, subseriatim punctatis, metasterno punctatissimo. Long. $3 \mathrm{~mm}$.

Ebenfalls dem St. ater und ebenso der vorigen Art sehr ähnlich und nahe verwandt; die Flügeldecken bedecken aber das Pygidium vollständig und die Hinterbrust ist sehr gedrängt, stark punktirt. In Bezug der Körperform stimmt diese Art mit der vorigen überein.

Miyanoshita, Mikuni-togè.

\section{Strongylus dorsalis:}

Hemisphaericus, subtilissime punctatus, nitidus, subtus cum antennis (clava fusca excepta) pedibusque ferrugineus, supra

„Wiener Entomologische Zeitung“ IV. (15. März 1885). Heft 3. 
niger, ore, capitis margine postico, prothoracis lateribus, his macula dorsali curvata utrinque et elytris macula subrotundata, leviter transversa, prope suturam ante medium sita, sanguineis. Long. $3.5 \mathrm{~mm}$.

Mit St. ornatus verwandt, aber durch andere Färbung des Halsschildes und dunklen Kopf zu unterscheiden. Der Halsschild ist schwarz, jederseits befindet sich eine blutrothe gebogene Makel, welche sich längs des Hinterrandes mit dem rothen Seirenrande verbindet.

Konosé, einige Stiicke.

\section{Strongylus excellens :}

Hemisphaericus, sat dense fortiter punctatus, nitidus, subtus cum pedibus ferrugineus, supra niger, antennarum basi, capite (fascia media transversa, obsoleta, nigra, excepta) prothoracis lateribus antice, fascia transversa, arcuata, in medio, scutello antice, elytra macula O-formi, dorsali, antice trilobata, postice extrorsum prolongata, sanguineis; elytris obsoletissime substriatis, punctis omnibus subaequalibus, pygidio subnudo, apice ferrugineo. Long. $4 \cdot 2 \mathrm{~mm}$.

Die O-förmige rothe Zeichnung der Flügeldecken entsendet 3 Aeste nach vorn und ist auch unten, gegen den Seitenrand zu ästig verlängert. Sie hat die Tendenz, in der Mitte der Aussenseite zu erlöschen.

Sapporo.

\section{Neopallodes n. gen.}

Gen. Pallodi similis, sed tibiae anticae nonunquam apice bidenticulatae, intermediae muticae, tarsi postici tibïs haud longiores, prothorax basi juxta scutellum utrinque emarginatus. Corpus magis convexum, fere semiglobosum.

\section{Neopallodes clavatus:}

Niger, nitidus, subtus cum antennis (clava fusca excepta) pedibusque testaceus, capite prothoracis lateribus late ferrugineis, capite prothoraceque subtiliter, elytris magis distincte subseriatim geminato-punctatis, antennarum clava magna. Long. $3 \cdot 2 \mathrm{~mm}$.

Schwarz, glänzend, die Unterseite, die Fühlergeissel und Beine röthlich gelb, der Kopf und die breiten Seiten des Halsschildes rostroth. Häufig ist die Umgegend des Schildchens pechbraun durchscheinend. Die braune Fühlerkeule ist so lang als das 2. bis 8. Fühlerglied zusammen. Kopf 
dicht, Halsschild viel feiner und weitläufiger punktirt, die Hinterwinkel des letzteren sind wenig grösser als rechte. Schildchen in der Mitte spärlich punktirt. Flügeldecken das rothgelbe Pygidium nicht bedeckend, stärker als der Halsschild punktirt, die Punkte paarweise, unregelmässig genähert und zu Längsreihen geordnet, die Nahtlinie ist leicht übersehbar, weil sie ganz dicht an die Naht gerïckt erscheint. Vorderschienen an der Spitze aussen mit 1 oder 2 Zähnchen. Hinterschienen am äusseren Apicalwinkel mit einem kleinen Dörnchen.

Diese Art ist vor allen anderen dieser Gattung durch die sehr grosse und entsprechend breite Fühlerkeule ausgezeichnet.

$\mathrm{Nara}$ und Kioto.

\section{Neopallodes inermis:}

Breviter ovalis, convexus, niger, nitidus, antennarum basi, pedibus, elytrorum fascia transversa basali indeterminata ferrugineis, capite prothoraceque dense punctatis, hoc valde transverso, coleopteris parum angustiore, antrorsum fortiter angustato, antice emarginato, basi prope scutellum utrinque subito sinuata, scutello nigro, parce punctato, elytris convexis, pygidium rufo-brunneum haud obtegentibus, striato-punctatis, interstitiis parce subtiliter, fere seriatim punctulatis, tibïs anticis extus apice rotundatis, inermibus. Long. $3 \mathrm{~mm}$.

Die Unterseite, namentlich der Bauch ist gewöhnlich braunroth. Die dunkle Fühlerkeule ist nicht so lang als das 2. bis 8. Fühlerglied zusammen. Von allen Arten durch die unbewehrten Vorderschienen abweichend.

Oyama bei Yokohama, am 25. Mai gefangen.

\section{Pallodes cyrtusoides :}

Breviter ovatus, testaceus, oculis nigris, clava antennarum subfusca; elytris punctatis, punctis vix evidenter seriatis. Long. $1 \cdot 8-2 \cdot 4 \mathrm{~mm}$.

Einfarbig gelbroth, Kopf und Halsschild höchst fein und ziemlich dicht, aber sehr undeutlich, Flügeldecken deutlicher punktirt, die Punktirung keine deutlichen Reihen bildend.

$\mathrm{Nagasaki}$; auch von Hiller in Japan gesammelt.

\section{Cryptarcha inhalita:}

Ovata, sat convexa, nitidula, subtiliter obscure puberula, pilis paulo longioribus, in elytris subseriatim dispositis fulvis,

„Wiener Entomologische Zeitung“ IV. (15. März 1885). Heft 3. 
intermixtis, brunneo-ferruginea, capite postice, prothoracis dorso, elytrorum maculis 3-4 valde indistinctis, subfuscis, antennis pedibusque testaceis; capite prothoraceque distincte, elytris magis dense et magis subtiliter punctatis; scutello minutissimo valde transverso. Long. $2 \cdot 5 \mathrm{~mm}$.

Von der Form der Cr. strigata, ähnlich behaart, rostbraun, der Scheitel, die Scheibe des Halsschildes und mehrere unbestimmt angedeutete Makeln auf den Flügeldecken (2 vor der Mitte und 1-2 vor der Spitze) dunkler braun. Von allen anderen Arten durch das schwer sichtbare, kleine, stark quere Schildchen leicht zu erkennen.

\section{Librodor ciavatus:}

Fukahori bei Nagasaki, 1 Expl., am 11. April 1881.

Oblongo-ovatus, parum convexus, subtus cum pygidio, antennarum basi tarsisque rufo-ferrugineus, supra niger, nitidus, prothorace transverso, basi utrinque sinuato, elytris maculis duabus, prima subbasali trilobata, altera transversa pone medium sita, aurantiacis, antennarum clava magna, subparallela, articulis duobus primis levissime transversis. Long. 5-6 $\mathrm{mm}$.

Von der Körperform einer länglichen Cryptarcha, lang eiförmig, nur etwas gewölbt, überall gleichmässig, ziemlich dicht punktirt, die Unterseite, das Pygidium, die Wurzel der Fühler und die Beine, meist aber nur deren Tarsen rostroth; oben schwarz, manchmal die schmalen Seitenränder des Halsschildes und der Hinterrand des Kopfes düster rostroth. Halsschild mindestens so breit als die Flügeldecken, quer, nach vorne verengt, die Hinterecken spitzig, die Basis doppelbuchtig, über der Ausbuchtung quer, grübchenartig gerandet. Schildchen dreieckig, an der Basis punktulirt. Flügeldecken ganz ähnlich wie bei unserem quadriguttatus Fbr. gezeichnet, die Zeichnung ist aber mehr gelbroth als gelb, und die hintere Querbinde hat nach hinten an der Naht einen schmalen Fortsatz. Die zwei ersten Glieder der auffällig grossen, gestreckten Fühlerkeule sind wenig breiter als lang, wodurch sich diese Art sofort von allen übrigen erkennen lässt.

Kiga, Usui-togè.

(Fortsetzung folgt.) 


\section{$2 \mathrm{BHL}$ Biodiversity Heritage Library}

Reitter, Edmund. 1885. "Die Nitiduliden Japans. Fortsetzung." Wiener entomologische Zeitung 4, 75-80. https://doi.org/10.5962/bhl.part.20099.

View This Item Online: https://www.biodiversitylibrary.org/item/44101

DOI: https://doi.org/10.5962/bhl.part.20099

Permalink: https://www.biodiversitylibrary.org/partpdf/20099

\section{Holding Institution}

Smithsonian Libraries

\section{Sponsored by}

Smithsonian

\section{Copyright \& Reuse}

Copyright Status: NOT_IN_COPYRIGHT

This document was created from content at the Biodiversity Heritage Library, the world's largest open access digital library for biodiversity literature and archives. Visit BHL at https://www.biodiversitylibrary.org. 